\begin{tabular}{|c|l|}
\hline Title & Particle like and fluid-like settling of a stratified suspension \\
\hline Author(s) & Harada, Shusaku; Mitsui, Takashi; Sato, Kodai \\
\hline Citation & $\begin{array}{l}\text { The European physical journal. E, Soft matter and biol ogical physics, 35, 1 } \\
\text { https://doi.org/10.1140/epje/2012-12001-6 }\end{array}$ \\
\hline Issue Date & 2012-01 \\
\hline Doc URL & http://hdl.handle.net/2115/48407 \\
\hline Rights & The original publication is available at www.eurphys. org \\
\hline Type & article (author version) \\
\hline File Information & EPJE,2012,35,1-6.pdf \\
\hline
\end{tabular}

Instructions for use 


\title{
Particle-like and Fluid-like Settling of a Stratified Suspension
}

\author{
Shusaku Harada ${ }^{a}$, Takashi Mitsui and Kodai Sato \\ Division of Sustainable Resources Engineering, Faculty of Engineering, Hokkaido University, N13-W8, Sapporo, Hokkaido, \\ 060-8628, Japan
}

Received: date / Revised version: date

\begin{abstract}
The gravitational settling of inhomogeneously suspended particles in fluid has been investigated. Of particular interest is whether collective or individual motion of particles is dominant during their settlings, i.e., whether the particles settle as a continuous suspension or they settle individually relative to surrounding fluid. We observed the settling of a stratified suspension which has the lower and upper concentration interfaces in quasi-two dimensional vessel. In some cases, the suspension behaves perfectly as a continuous fluid and the motion of the constituent particle is subject to bulk flow caused by the interfacial instability. In other cases, the particle behaves individually relative to surrounding fluid. The existence of concentration interface plays a significant role in these extreme behaviors of suspension. The transition from the collective to individual behaviors can be predicted quantitatively by a parameter which expresses the border resolution of concentration interface.
\end{abstract}

PACS. 47.61.Jd Multiphase flows

\section{Introduction}

The gravitational settling of suspended particles in fluid can be found not only in natural phenomena but also in engineering processes such as accumulation or separation of particulate materials. If particles are suspended homogeneously in fluid and their density is higher than that of the fluid, the particles move downward relative to the surrounding fluid and the variation of the concentration evolves one-dimensionally along the gravitational direction [1]. The settling velocity of suspended particle varies with local concentration due to hydrodynamic interactions with their surrounding particles [2]. In general, hydrodynamic interactions between particles are long-ranged under conditions of low particle Reynolds number, since the disturbed flow by the motion of individual particles decays inversely proportional to the interparticle distance [3]. Particularly the settling motion of fine particles is greatly influenced by the hydrodynamic interaction because of their small inertia (low Stokes number) in addition to low particle Reynolds number.

In the gravitational settling of fine particles, not only does the hydrodynamic interaction affect the mean settling velocity but also it induces the velocity variance of suspended particles. The resultant fluctuating motion brings about the diffusion of settling particles, which is called hydrodynamic diffusion. A lot of experimental and theoretical studies concerned with the hydrodynamic diffusion have been performed and have been found that the diffusivity of particles has a strong anisotropy and is influ-

\footnotetext{
a e-mail: harada@eng.hokudai.ac.jp
}

enced by many factors such as the particle size, the vessel size and so on [4-10].

In case that particles are suspended in fluid with spatial concentration variations, the settling behavior is more complicated. In particular, if the suspension has a concentration interface, which is apparent interface between suspension and pure liquid, the relative motion of particle is much more enhanced and it brings about the macroscopic motion of suspension. One typical example is a well-known Boycott effect [11]. When the suspended particles settle in an inclined tube, the settling velocity is much larger than that in a vertical tube. One of the reasons of such a rapid settling is fluid convection caused by the concentration gradient. This convection effect is enhanced most if the system has concentration interface. The other example is falling clouds of particles in liquid. Some researchers have found the interesting motion of particle clouds such as torus formation, cloud breakup or leakage of particles [12-15]. These motions occur under the influence of the concentration interface which borders the particle clouds.

A horizontally-stratified suspension is also a typical example of inhomogeneous suspensions with concentration interfaces. In a stratified suspension with the positive concentration gradient against the gravity, the interfacial instability occurs at the suspension-fluid boundary and it brings about a lateral variation of the concentration [1923]. In this case, the suspension behaves as an immiscible fluid even though there is no distinct border with pure fluid. The motion of suspended particles is subject to bulk flow caused by the instability and the settling velocity becomes far from that of an isolated particle. 
The purpose of this study is to investigate whether collective or individual motion of particle is dominant during the settling of an inhomogeneous suspension, and what decides these contrastive behaviors. We have investigated the settling of a stratified suspension which has both the upper and lower interfaces of concentration in quasi-two dimensional vessel. We measured the settling velocity of particles near the concentration interface and examined whether the particles settle collectively as a continuous fluid or as individual bodies relative to surrounding fluid.

\section{Experimental method}

Figure 1 shows the schematic diagram of experimental apparatus. The test cell is a quasi-two dimensional vessel with a height $L=240 \mathrm{~mm}$ and a width $W=100 \mathrm{~mm}$. The vessel is made by acrylic plates except for the front glass. The vessel thickness $D$ is adjustable from 3 to $12 \mathrm{~mm}$. There are two slits on the back side of the vessel for stainless steel blade insertion. The thickness of the blade is $0.5 \mathrm{~mm}$ and the distance between two blades $L_{S}$ is $19.5 \mathrm{~mm}$. The lower blade separates the lower pure fluid and suspension, while the upper blade separates the upper fluidsuspension. Therefore the distance between blades corresponds to the initial height of the stratified suspension.

The experimental procedure is as follows. At first pure fluid is filled into the lower part of the vessel and then the lower part is closed by the lower blade. Next suspension is poured into the vessel above the lower blade until the surface reaches the position of the upper blade. Finally the upper blade is put into the vessel and the pure fluid is filled above it. Consequently, a stratified suspension is held between two blades. To begin an experiment, the stainless steel blades are removed backward simultaneously. The settling behavior of particles near the center of the vessel is recorded by a digital video camera.

The suspension contains silicone oil and glass or polystyrene particles. The diameter of particle is $d_{p}=30$ or $100 \mu \mathrm{m}$ for glass and $d_{p}=550$ or $800 \mu \mathrm{m}$ for polystyrene.

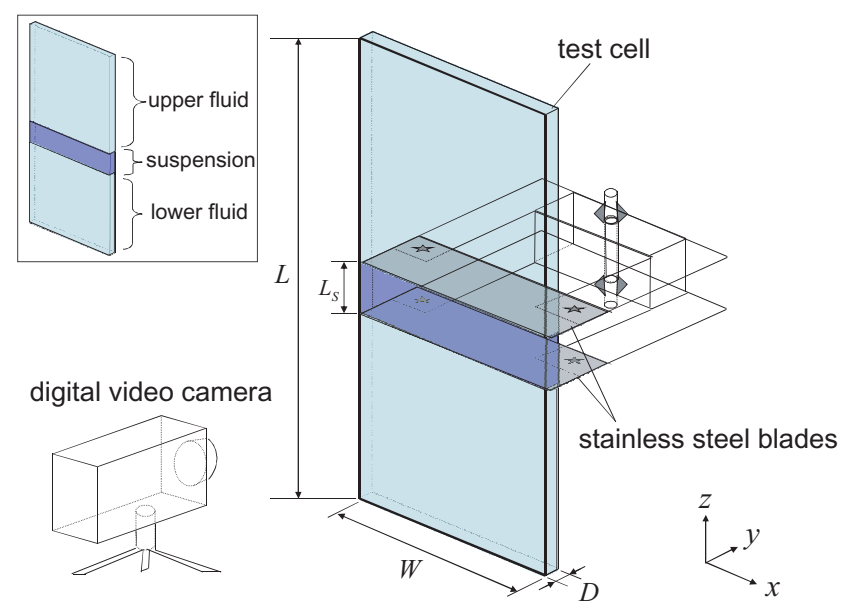

Fig. 1. Schematic diagram of experimental system
The mass density of glass and polystyrene is $\rho_{p}=2500$ and $1050 \mathrm{~kg} / \mathrm{m}^{3}$ respectively. The suspension is mixed by stirring for several hours and is deaerated at constant temperature $\left(22 \pm 1 \mathrm{C}^{\circ}\right)$. The silicone oil (density $\rho_{f}=$ $972 \mathrm{~kg} / \mathrm{m}^{3}$, viscosity $\mu_{f}=0.97$ or $1.94 \mathrm{~Pa} \cdot \mathrm{s}$ ) is used for making suspension. The constituent fluid of the suspension is the same as the upper and lower fluid. Therefore the stratified suspension can be interpreted as partially suspended particles in a pure fluid.

As described below, the Stokes settling velocity $U_{0}$ on our experimental condition is in the range of $10^{-4}-10^{-2}$ $\mathrm{mm} / \mathrm{s}$. In order to verify whether the uniformity of the suspension is kept during the experimental setting, we estimate the settling distance of particles during the setting. The period from the filling of suspension to the removal of blades is a few minutes. We can estimate the maximum settling distance of particle during this period at a few particle size in all conditions. Therefore we consider the uniformity of the suspension to be maintained during preparation.

\section{Results and discussion}

\subsection{Settling behavior of stratified suspension}

Experiments were conducted by various combinations of particle and fluid in addition to changing particle concentration $\phi$ and vessel thickness $D$. Table 1 shows the conditions of each experiment, e.g. the particle diameter $d_{p}$, the particle volumetric concentration $\phi$, the fluid viscosity $\mu_{f}$ and the vessel thickness $D$. The particle density $\rho_{p}$ and fluid density $\rho_{f}$ are already described in the above section. The Stokes settling velocity for a single particle $U_{0}$ is also shown in the Table 1 . The particle Reynolds number $R e=\rho_{f} d_{p} U_{0} / \mu_{f}$ is less than $10^{-4}$ in all cases.

Figure 2 shows typical examples of settling behavior of suspension. As seen in Fig.2, unevenness of the lower interface develops with time, while the upper interface remains almost flat. The flat upper interface is due to a self-sharpening effect which results from the dependency of the settling velocity on the local particle concentration [1]. The unevenness of the lower interface is obviously a gravity-induced instability (Rayleigh-Taylor instability). Some researchers have reported that such an instability occurs at the suspension-fluid boundary even though there is no distinct interface between them [19-23].

In case of small particles (Fig.2a), the suspension behaves like immiscible fluid and finger-like perturbations clearly can be found. It should be emphasized again that the system considered here is partially suspended particles in pure fluid since the suspension is made of the same fluid as the upper and lower ones. Therefore there is no defined border between suspension and fluid. We call such a continuum behavior a fluid-like settling of suspension. On the other hand, in case of large particles (Fig.2c), the suspension-fluid interface is less distinct and the suspended particles settle individually. We call this settling behavior a particle-like settling of suspension. The settling velocity of constituent particle is greatly influenced 
Table 1. Physical properties of particle and fluid

\begin{tabular}{|c|c|c|c|c|c|c|c|}
\hline & & $d_{p}(\mu \mathrm{m})$ & $\phi$ & $\mu_{f}(\mathrm{~Pa} \cdot \mathrm{s})$ & $D(\mathrm{~mm})$ & $U_{0}(\mathrm{~mm} / \mathrm{s})$ & $H$ \\
\hline$(\mathrm{A})$ & glass particle & 30 & 0.03 & 1.94 & 8 & $3.85 \times 10^{-4}$ & 0.00524 \\
\hline (B) & polystylene particle & 550 & 0.1 & 0.97 & 8 & $1.34 \times 10^{-2}$ & 0.0642 \\
\hline (C) & polystylene particle & 800 & 0.01 & 1.94 & 12 & $1.40 \times 10^{-2}$ & 0.134 \\
\hline (D) & glass particle & 30 & 0.03 & 1.94 & 5 & $3.85 \times 10^{-4}$ & 0.00837 \\
\hline$(\mathrm{E})$ & glass particle & 30 & 0.03 & 1.94 & 3 & $3.85 \times 10^{-4}$ & 0.0140 \\
\hline$(\mathrm{F})$ & glass particle & 100 & 0.07 & 1.94 & 5 & $4.28 \times 10^{-3}$ & 0.0210 \\
\hline (G) & polystylene particle & 800 & 0.005 & 0.97 & 8 & $2.84 \times 10^{-2}$ & 0.254 \\
\hline (H) & glass particle & 30 & 0.01 & 1.94 & 5 & $3.85 \times 10^{-4}$ & 0.0121 \\
\hline (I) & glass particle & 30 & 0.04 & 1.94 & 5 & $3.85 \times 10^{-4}$ & 0.00761 \\
\hline$(\mathrm{J})$ & glass particle & 30 & 0.05 & 1.94 & 5 & $3.85 \times 10^{-4}$ & 0.00706 \\
\hline$(\mathrm{K})$ & glass particle & 100 & 0.02 & 1.94 & 5 & $4.28 \times 10^{-3}$ & 0.0320 \\
\hline$(\mathrm{L})$ & glass particle & 100 & 0.03 & 1.94 & 5 & $4.28 \times 10^{-3}$ & 0.0279 \\
\hline (M) & glass particle & 100 & 0.04 & 1.94 & 5 & $4.28 \times 10^{-3}$ & 0.0254 \\
\hline$(\mathrm{N})$ & glass particle & 100 & 0.05 & 1.94 & 5 & $4.28 \times 10^{-3}$ & 0.0235 \\
\hline
\end{tabular}
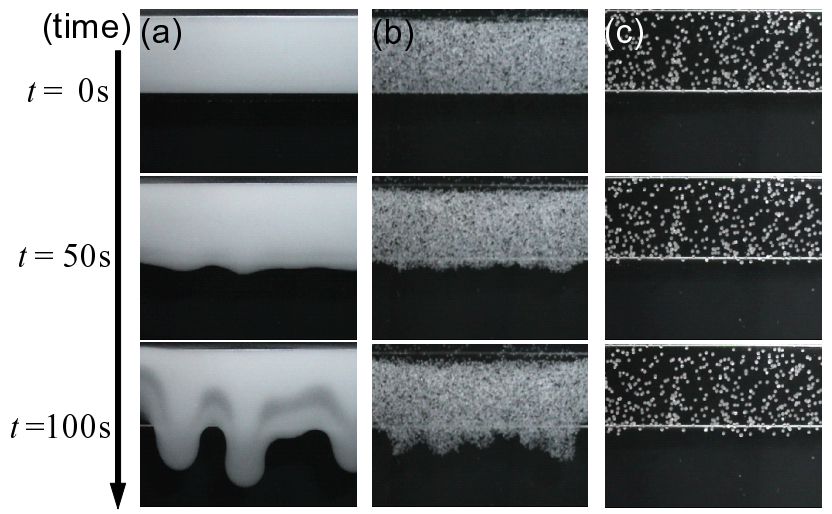

Fig. 2. Settling behaviors of stratified suspension in quasi-two dimensional vessel; (a) glass particles $\left(d_{p}=30 \mu \mathrm{m}, \phi=0.03\right.$, $D=8 \mathrm{~mm})$, (b) polystyrene particles $\left(d_{p}=550 \mu \mathrm{m}, \phi=0.1\right.$, $D=8 \mathrm{~mm})$ and (c) polystyrene particles $\left(d_{p}=800 \mu \mathrm{m}, \phi=\right.$ $0.01, D=12 \mathrm{~mm}$ ). Detailed conditions are found in Table 1 (conditions (A), (B) and (C) respectively).

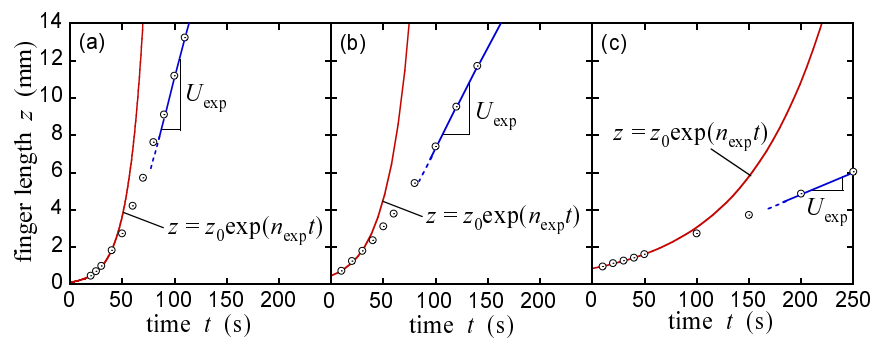

Fig. 3. Change in average length of fingers with time. (a)-(c) correspond to those in Fig.2.

by these behaviors. It is visually found that the settling velocity on case (a) is hundreds of times larger than the Stokes settling velocity $\left(U_{0}=3.85 \times 10^{-4} \mathrm{~mm} / \mathrm{s}\right)$. In addition, the settling velocity on case (b) is much faster than case (c), although Stokes settling velocity on both cases are almost the same $\left(U_{0}=1.34 \times 10^{-2}\right.$ and $1.40 \times 10^{-2}$ $\mathrm{mm} / \mathrm{s}$ respectively).
For quantitative discussions of the settling velocity, the growth of finger-like perturbation (finger) was measured by image analysis. Figure 3 shows the change in the average length of fingers with time under the same conditions of Fig.2. The finger length was defined as the distance between the position of the lower blade (initial position of the lower boundary of suspension) and that of the undermost particle in each finger. In case that the fingers are ill-defined, once we recognized them from pictures at which time the fingers fully grow up, and then measured their length for each instant of time. w The fingers grow exponentially at the beginning and then the growth velocity becomes constant in all cases. The exponential growth of fingers is characteristic of the Rayleigh-Taylor instability of immiscible fluids and the finger length is defined as $z=z_{0} \exp (n t)$, where $n$ is the growth rate. We can define two experimental values which express the settling speed of particles. One is the initial growth rate of fingers $n_{\exp }$, which is obtained from the fitting of exponential function to the beginning of the experimental results. The other is the succeeding settling velocity $U_{\text {exp }}$, which is defined as the time rate of change in finger length at a later stage as shown in Fig.3.

If the suspension behavior is perfectly particle-like, the succeeding growth velocity $U_{\text {exp }}$ is expected to be in the same order of magnitude as the Stokes settling velocity $U_{0}$. We compare the experimental results from this point of view. The ratio $U_{\text {exp }} / U_{0}$ on cases (a)-(c) is 535, 8.04 and 1.91 respectively. This implies that $U_{\exp } / U_{0}$ is an indicator of independent settling of particles. That is, the settling behavior on case (c) is more particle-like than case (a). More quantitative discussions are given later.

\subsection{Instabilities of fluid-like settling of suspension}

In order to understand the fluid-like settling of suspension shown in Fig.2(a), the experimental results are compared to the theoretical results for immiscible fluids. Some researchers have developed the theory of gravitational instability of miscible or immiscible fluids in a Hele-Shaw 


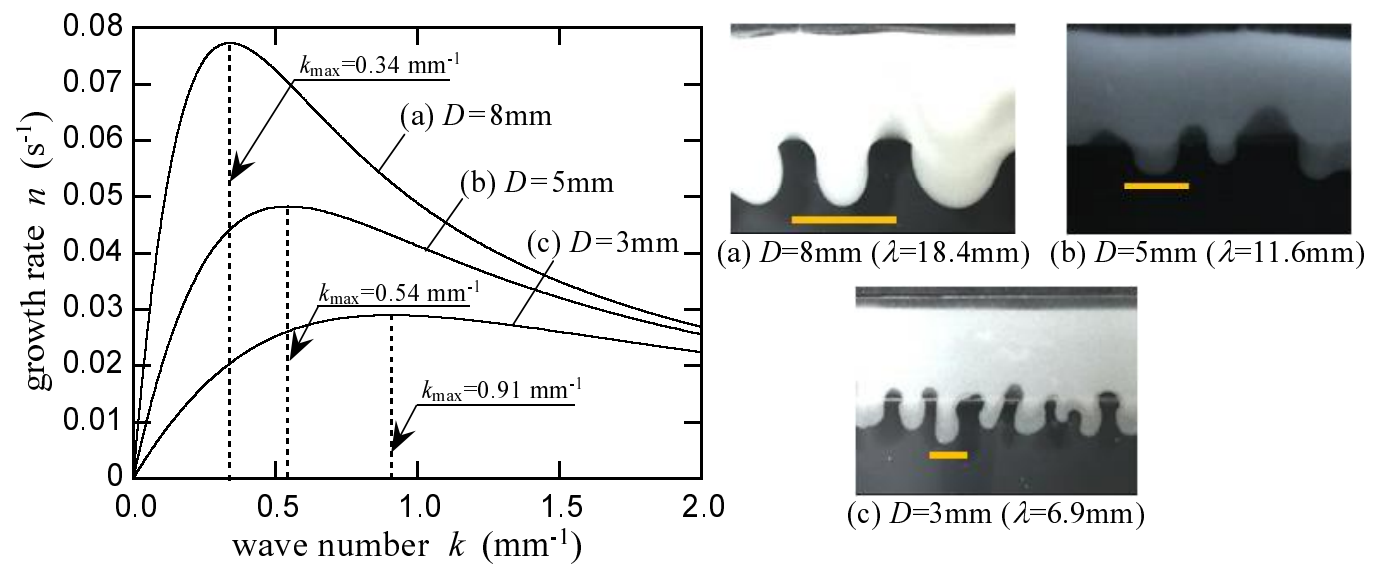

Fig. 4. Dominant wave number $k_{\max }$ calculated by linear stability analysis and corresponding wave length $\lambda=2 \pi / k_{\max }$ for glass particle-silicone oil suspension $\left(d_{p}=30 \mu \mathrm{m}, \phi=0.03\right)$ with various vessel thickness $D$. Detailed conditions are found in Table 1 (conditions (A), (D) and (E) respectively).

cell [16-18]. Völtz et al. [19-21] have studied systematically the gravitational settling of fine particles in narrow channel. They have reported that the Rayleigh-Taylor instability at suspension-fluid interface could be predicted by linear stability analysis of immiscible viscous fluids. We also performed the stability analysis by the method proposed by them [20]. The suspension is assumed to be a continuous fluid having apparent density and viscosity. The interfacial tension between suspension and pure fluid is set to be zero. The system has finite space in gap direction $(y)$ while it is infinite in lateral $(x)$ and vertical $(z)$ directions. On the assumption that the vessel has narrow gap, the velocity in $y$ direction is set to be zero and the velocity and density fluctuation has parabolic Poiseuille profiles as $u_{i}=\hat{u}_{i}(z) \exp (i k x+n t) \zeta(y), \rho=\bar{\rho}(z)+\hat{\rho}(z) \exp (i k x+$ $n t) \zeta(y)[20,24]$ where $k$ is the wave number of disturbance and $n$ is the growth rate. $\zeta(y)$ indicates a parabolic function defined by $\zeta(y)=6\left(D^{2} / 4-y^{2}\right) / D^{2}$. Substituting these equations to Stokes equation (linearized NavierStokes equation) and continuity equation and averaging over $y$ direction, we obtain the relation between $k$ and $n$;

$$
\begin{aligned}
& -\left[\frac{g k}{n^{2}}\left(\alpha_{1}-\alpha_{2}\right)+\left(\beta_{1}+\beta_{2}\right)\right]\left[\beta_{1} q_{1}+\beta_{2} q_{2}-k\left(\beta_{1}+\beta_{2}\right)\right] \\
& -4 k \beta_{1} \beta_{2}+\frac{4 k^{2}}{n}\left(\alpha_{1} \bar{\nu}_{1}-\alpha_{2} \bar{\nu}_{2}\right)\left[\beta_{1} q_{1}-\beta_{2} q_{2}+k\left(\beta_{1}-\beta_{2}\right)\right] \\
& +\frac{4 k^{3}}{n^{2}}\left(\alpha_{1} \bar{\nu}_{1}-\alpha_{2} \bar{\nu}_{2}\right)^{2}\left(q_{1}-k\right)\left(q_{2}-k\right)=0
\end{aligned}
$$

where $\bar{\nu}$ is kinematic viscosity and subscripts 1 and 2 indicate the lower and the upper fluid respectively. $\alpha, \beta$ and $q$ are defined as $\alpha_{1}=\bar{\rho}_{1} /\left(\bar{\rho}_{1}+\bar{\rho}_{2}\right), \alpha_{2}=\bar{\rho}_{2} /\left(\bar{\rho}_{1}+\bar{\rho}_{2}\right), \beta_{1}=$ $\alpha_{1}\left(1+\bar{\nu}_{1} \eta / n\right), \beta_{2}=\alpha_{2}\left(1+\bar{\nu}_{2} \eta / n\right), q_{1}=\sqrt{n / \bar{\nu}_{1}+k^{2}+\eta}$, $q_{2}=\sqrt{n / \bar{\nu}_{2}+k^{2}+\eta}$, and $\eta=\partial^{2} \zeta / \partial y^{2}=12 / D^{2}$. If the vessel thickness $D$ approaches infinity, $\beta$ tends to zero and consequently Eq.(1) is close to well-known $k$ - $n$ relation for infinite space described in Ref.[25]. For suspension-pure fluid case, we set $\bar{\rho}_{1}, \bar{\nu}_{1}$ (lower fluid) to be properties of pure fluid $\rho_{f}, \nu_{f}$ and set $\bar{\rho}_{2}, \bar{\nu}_{2}$ (upper fluid) to be apparent properties of suspension $\rho_{s}=(1-\phi) \rho_{f}+\phi \rho_{p}$ and $\mu_{s}=(1+2.5 \phi) \mu_{f}$. Calculating Eq.(1) by Newton-Raphson method, the growth rate $n$ for a given $k$ is obtained.

Figure 4 shows the relation between the wave number $k$ and the growth rate $n$ calculated by Eq.(1) for glass particle-silicone oil suspension $\left(d_{p}=30 \mu \mathrm{m}, \phi=0.03\right.$ and $\left.\mu_{f}=1.94 \mathrm{~Pa} \cdot \mathrm{s}\right)$ with various vessel thickness $D$. The theoretical analysis indicates that the wave number of the fastest-growing disturbance $k_{\max }$ depends on $D$. Fig. 4 also shows the experimental pictures corresponding to the theoretical conditions. The solid line in each picture indicates the wave length of the fastest-growing disturbance $\lambda=2 \pi / k_{\max }$ predicted by the theoretical results. The theoretical predictions are in quantitative agreement with the finger width observed in the experiment. These results indicate that the disturbance with the largest growth rate survives among various disturbances of initial suspensionfluid interfaces.

As reported by Völtz et al. [20], if the particle size is adequately small, the suspension behaves perfectly fluid-like and the growth rate obtained by experiment $n_{\exp }$ would be close to the theoretical growth rate of the fastest-growing disturbance $n_{\text {theo }}$. The ratio $n_{\exp } / n_{\text {theo }}$ for case (a)-(c) shown in Fig.2 is $0.87,1.88$ and 6.27 respectively. This implies that $n_{\exp } / n_{\text {theo }}$ is an indicator of fluid-like settling, i.e., the settling behavior on case (a) is more fluid-like than case $(c)$.

\subsection{Transition from fluid-like to particle-like settling}

Here we pay attention to the transition from such fluidlike behaviors of suspension to individual behaviors of constituent particles. In order to find what decides the settling behavior of suspension as fluid-like or particle-like, we consider the parameter which describes the transition 
from fluid-like to particle-like settling of suspension. Thi parameter is obviously related to particle density $\rho_{p}$, par ticle diameter $d_{p}$, liquid density $\rho_{f}$, liquid viscosity $\mu_{f}$ particle concentration $\phi$ and vessel thickness $D$.

Since the visual difference between two extreme be haviors (Fig.2(a) and (c)) is the sharpness of interface, w propose a parameter which expresses the border resolutio: of concentration interface;

$$
H=l / \lambda
$$

where $l$ is the average distance between particles in suspension and $\lambda$ is the wave length of the fastest-growing perturbation (finger width), which can be obtained from linear stability analysis. Figure 5 indicates the physical meaning of Eq.(2). It can be found that the parameter expresses the border resolution of concentration interface. That is, when $H$ is large, the particles in suspension cannot form the finger clearly. The average distance between particles $l$ is obviously a function of $d_{p}$ and $\phi$ and approximately $l \sim d_{p} / \phi^{1 / 3}$. On the other hand, as found in Eq.(1), the wave length $\lambda$ is a function of $\rho_{p}, \rho_{f}, \mu_{f}, \phi$ and $D$. Consequently $H$ contains all six properties described above.

Figure 6 shows $n_{\exp } / n_{\text {theo }}$ and $U_{\text {exp }} / U_{0}$ obtained from 14 experiments with variation of the fluid and particle properties and also the vessel thickness. The horizontal axis is set to be the parameter $H$ given by Eq.(2). As can be seen in Fig.6, $n_{\exp } / n_{\text {theo }}$ is around unity, while $U_{\exp } / U_{0}$ indicates larger value for $H<0.03$. These results indicate that the suspension perfectly behaves like fluid in this region. On the contrary, $U_{\exp } / U_{0}$ is around unity and $n_{\exp } / n_{\text {theo }}$ is apart from unity for $H>0.2$. These results suggest the particle-like settling of suspension for larger $H$. We can classify the settling behaviors of suspension as fluid-like, particle-like settlings and their transition by $H$. Figure 6 also indicates some pictures of the settling behavior of suspension. We can understand visually that this parameter is reasonable to classify the settling behavior.

The similar collective motion of particulate suspension has been studied on the settling of particle clouds in viscous fluid. Some researchers pointed out that the collective motion of particle clouds can be explained by the swarm of Stokeslet [12-14]. They found that the particle clouds behaves collectively when the number density of particle (Stokeslet), which describes the discretization inside the clouds, is sufficiently large. If $l$ is expressed by number density of particles $N=6 \phi / \pi d_{p}^{3}$, Eq.(2) is written as $H=(6 / \pi)^{1 / 3} \lambda^{-1} N^{-1 / 3}$. Therefore $H$ is interpreted as the discretization by the number density $N$ to the given lengthscale $\lambda$, which is consistent with the previous studies. One more interesting similarity to previous study is about the settling velocity. Adachi [15] reported that the particle clouds settle as one body when the ratio of the settling velocity to that of an isolated particle is larger than 16. This can be interpreted as one aspect of the threshold for collective motion of clouds. As found in Fig.6, the settling velocity $U_{\exp } / U_{0}$ is larger than 15 in the fluid-like region. These thresholds are quite similar even though the

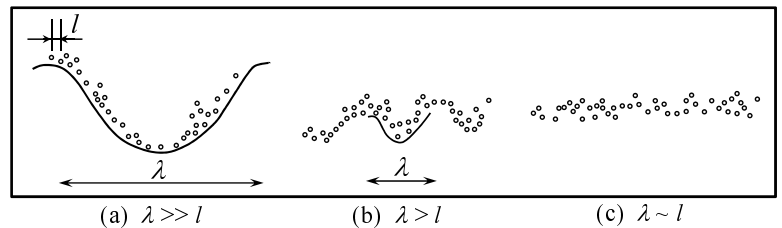

Fig. 5. Relation between wave length of interfacial perturbation and average distance between particles.

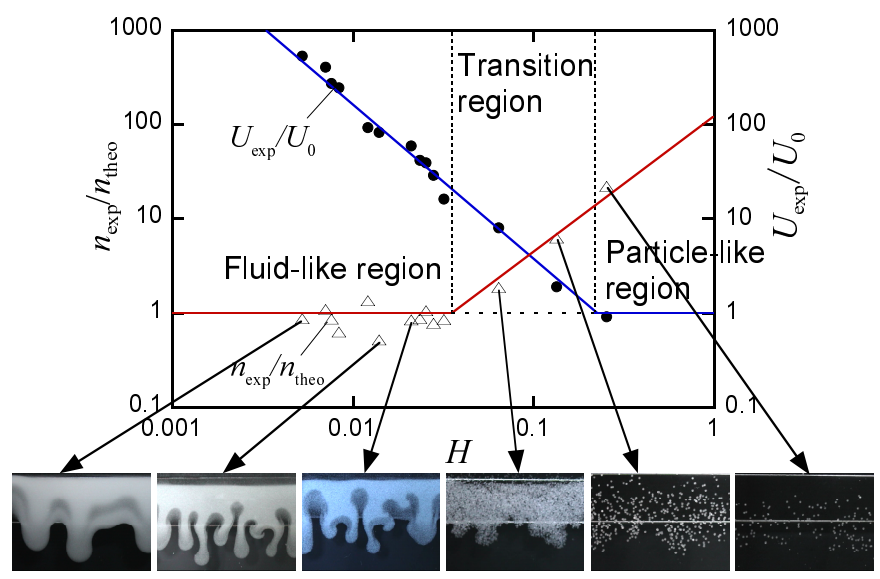

Fig. 6. Transition from fluid-like to particle-like behaviors of suspension by changing border resolution of concentration interface. Images correspond to conditions $(\mathrm{A}),(\mathrm{E}),(\mathrm{F}),(\mathrm{B}),(\mathrm{C})$ and $(G)$ starting from the left.

system studied here is considerably different from his system.

\section{Conclusions}

The gravitational settling of inhomogeneous suspensions in quasi two-dimensional vessel was investigated experimentally. We have examined whether the suspension behave as a particle assembly (particle-like settling) or as a continuous fluid (fluid-like settling). The results shown here indicate that sometimes the suspension behaves perfectly as a continuous fluid, while in other times, the particle behaves individually relative to surrounding fluid. The transition of these contrastive behaviors is determined by the border resolution of concentration interface. These results may provide important insight into the collective motion of particulate suspension.

This work has been partly supported by the Grant-in-Aid for Scientific Research (C) 23560178, Japan Society for the Promotion of Science.

\section{References}

1. G. J. Kynch, Trans. Faraday Soc. 48, 166 (1952). 
2. R.H. Davis, A. Acrivos, Ann. Rev. Fluid Mech. 17, 91 (1985).

3. J. Happel, H. Brenner, Low Reynolds number hydrodynamics: with special applications to particulate media (Kluwer Academic Publishers, Dordrecht, 1973)

4. S. Lee, Y. Jang, C. Choi, T. Lee, Phys. Fluids, A4, 2601 (1992).

5. J.-Z. Xue, E. Herbolzheimer, M. A. Rutgers, W. B. Russel, P. M. Chaikin, Phys. Rev. Lett. 69, 1715 (1992).

6. H. Nicolai, E. Guazzelli, Phys. Fluids 15, 1305 (2003).

7. H. Nicolai, B. Herzhaft, E. J. Hinch, L. Oger, E. Guazzelli, Phys. Fluids, 7, 12 (1995).

8. J. Martin, N. Rakotomalala, D. Salin, Phys. Rev. Lett. 74, 1347 (1995).

9. R. H. Davis, J. Fluid Mech. 310, 325 (1996).

10. P. J. Mucha, M. P. Brenner, Phys. Fluids 7, 3 (1995).

11. E. Guyon, J.-P. Hulin, L. Petit, C. D. Mitescu, Physical Hydrodynamics (Oxford University Press, New York, 2001)

12. J. M. Nitche, G. K. Batchelor, J. Fluid Mech. 340, 161 (1997).

13. G. Machu, W. Meile, L. C. Nitche, U. Schaflinger, J. Fluid Mech. 447, 299 (2001).

14. B. Metzger, M. Nicolas, E. Guazzelli, J. Fluid Mech. 580, 283 (2007).

15. K. Adachi, S. Kiriyama, N. Yoshioka, Chem. Eng. Sci. 33 115 (1978)

16. J. Fernandez, P. Kurowski, L. Limat, P. Petitjeans, Phys. Fluids 13, 3120 (2001).

17. J. Fernandez, P. Kurowski, P. Petitjeans, E. Meiburg, J. Fluid Mech. 451, 239 (2002).

18. P. Carlès, Z. Huang, G. Carbone, C. Rosenblatt, Phys. Rev. Lett. 96, 104501 (2006).

19. C. Völtz, M. Schröter, G. Iori, A. Betat, A. Lange, A. Engel, I. Rehberg, Phys. Rep. 337, 117 (2000).

20. C. Völtz, W. Pesch, I. Rehberg, Phys. Rev. E 65, 011404 (2001).

21. C. Völtz, Phys. Rev. E 68, 021408 (2003).

22. F. Blanchette, J. W. M. Bush, Phys. Fluids 17, 073302 (2005).

23. H. Michioka, I. Sumita, Geophys. Res. Lett. 32, L03309 (2005).

24. J. Huang, B. F. Edwards, Phys. Rev. E 54, 2620 (1996).

25. S. Chandrasekhar, Hydrodynamic and Hydromagnetic Stability (Dover Publications, New York, 1981) 